\title{
CARGAS ELÉTRICAS ESTRUTURAIS E VARIÁVEIS DE SOLOS TROPICAIS ALTAMENTE INTEMPERIZADOS ${ }^{(1)}$
}

\author{
Oscarlina Lucia dos Santos Weber ${ }^{(2)}$, José Carlos Chitolina ${ }^{(3)}$, Otávio \\ Antonio de Camargo ${ }^{(4)}$ \& Luis Reynaldo Ferracciu Alleoni ${ }^{(5)}$
}

\begin{abstract}
RESUMO
Os solos tropicais altamente intemperizados apresentam teor significativo de colóides com carga elétrica variável. Entretanto, são poucas as referências em relação à quantificação destas cargas, principalmente em solos ácricos, que representam o extremo na escala de intemperismo. Neste estudo, foram determinadas as cargas permanentes e as variáveis de dois Latossolos Vermelhos acriférricos, um Latossolo Amarelo ácrico e um Latossolo Amarelo acriférrico, que foram comparados a um Nitossolo Vermelho eutroférrico, com carga predominantemente permanente. As amostras foram investigadas pelo método da adsorção do íon césio $\left(\mathrm{Cs}^{+}\right)$, que mede a carga estrutural permanente $\left(\sigma_{\mathrm{o}}\right)$ e baseia-se na preferência do $\mathrm{Cs}^{+}$sobre o $\mathrm{Li}^{+}$na superfície da siloxana de grupos de superfície ionizáveis de menor seletividade ao íon $\mathrm{Cs}^{+}$. A carga variável representou mais que $50 \%$ da carga total dos solos estudados. Dois dos quatro Latossolos com propriedades ácricas exibiram quantidade significativa de carga permanente, provavelmente em razão da presença de vermiculita com hidróxi entrecamadas e clorita. A quantidade de carga permanente apresentada pelo Nitossolo foi até cinco vezes maior se comparada à dos Latossolos, o que pode ser atribuído à diferença na constituição mineralógica. O método da adsorção de Cs foi capaz de identificar teores significativos de carga permanente estrutural, mesmo em solos com baixo teor de minerais 2:1.
\end{abstract}

Termos de indexação: césio, mineralogia, troca iônica.

\footnotetext{
(1) Parte da Tese de Doutorado da primeira autora, apresentada ao Programa de Pós-Graduação em Solos e Nutrição de Plantas da Escola Superior de Agricultura "Luiz de Queiroz" - ESALQ/USP. Recebido para publicação em e aprovado em

(2) Pesquisador Científico do Departamento de Solos e Engenharia Rural, Universidade Federal do Mato Grosso - UFMT/FAMEV. Av. Fernando Corrêa da Costa s/n, CEP 78060-900 Cuiabá (MT). E-mail: oscsan@uol.com.br

(3) Professor da Escola de Engenharia de Piracicaba - EEP. R. Monsenhor Martinho Salgot 560, CEP 13414-900 Piracicaba (SP). Email: jcchitolina@yahoo.com.br

(4) Pesquisador Científico do Centro de Solos e Recursos Agroambientais, Instituto Agronômico de Campinas - IAC. Caixa Postal 28, CEP 13010-970 Campinas (SP). Bolsista do CNPq. E-mail: ocamargo@iac.sp.gov.br

(5) Professor Associado do Departamento de Solos e Nutrição de Plantas. Escola Superior de Agricultura "Luiz de Queiroz" - ESALQ/ USP. Caixa Postal 09, CEP 13418-900 Piracicaba (SP). Bolsista do CNPq. E-mail: lrfalleo@esalq.usp.br
} 


\title{
SUMMARY: STRUCTURAL AND VARIABLE ELECTRIC CHARGES OF HIGHLY WEATHERED TROPICAL SOILS
}

\begin{abstract}
Highly weathered tropical soils present high amount of colloids with variable electrical charge. However, there are few references related to the quantification of such charges, mainly in soils with acric attributes, which represent one of the extremes in the weathering scale. In this study permanent and variable charges were determined in four Oxisols and compared to an Alfisol with predominantly permanent charge. Samples were investigated

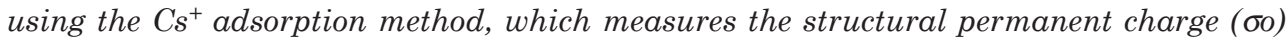
and is based on the preference of $\mathrm{Cs}^{+}$over $\mathrm{Li}^{+}$in the siloxane surface sites and the lower selectivity of ionizable surface group for the cesium ion. The variable charge represented more than $50 \%$ of the total charge of the evaluated soil samples. Two of the four Oxisols with acric properties had a significant permanent structural charge, probably due to small quantities of aluminum hydroxy-interlayer vermiculite and chlorite. The amount of permanent structural charges presented by the Alfisol was five times higher as compared to the Oxisols, which may be attributed to their mineral composition differences. The cesium adsorption method was able to quantify significant amounts of structural charges even in soils with a low amount of 2:1 clay minerals.
\end{abstract}

Index terms: cesium, electrochemical, ionic exchange.

\section{INTRODUÇÃO}

A maioria das reações que ocorrem na fração mineral da argila e na matéria orgânica coloidal, tais como: a adsorção e a dessorção de íons e a dispersão e floculação de colóides, são fenômenos de superfície. $\mathrm{O}$ entendimento da quantidade e da distribuição de cargas na superfície dos colóides é fundamental para explicar o comportamento das partículas coloidais, os atributos físicos e químicos do solo, bem como para recomendar o manejo dos solos das regiões dos trópicos úmidos e entender a evolução deles (Uehara, 1988).

As cargas elétricas nos colóides dos solos são didaticamente divididas em estruturais ou permanentes e variáveis. As permanentes desenvolvem-se na superfície da partícula sólida do solo por substituição iônica, enquanto as variáveis, por meio de dissociação e associação de prótons $\left(\mathrm{H}^{+}\right)$ (protonação/deprotonação) e adsorção específica de cátions e ânions (Stumm \& Morgan, 1981).

A fração argila dos solos das regiões de clima tropical e subtropical, por apresentar avançado grau de intemperização, é dominada por argilominerais do tipo 1:1, predominantemente caulinita. Este mineral, principal e virtualmente o único mineral silicatado em teor expressivo, e os óxidos e hidróxidos de Fe e de $\mathrm{Al}$ (Fontes et al., 2001) conferem superfície com carga elétrica variável nesses ambientes. É de se esperar que esses solos, principalmente nas camadas subsuperficiais, tenham baixíssimas quantidades de carga estrutural, à medida que avança o processo de intemperização, o que afeta a fertilidade do solo e a produtividade das culturas, como a da cana-de-açúcar (Landell et al., 2003).
Os solos ácricos são importantes do ponto de vista geográfico e econômico, uma vez que só em São Paulo totalizam aproximadamente 170.000 ha que assentam uma agricultura altamente tecnificada. Além disso, são importantes do ponto de vista taxonômico, visto que representam o extremo na escala de intemperização. Entretanto, seu comportamento eletroquímico, em relação à magnitude das cargas variáveis e estruturais, e sua relação com atributos físicos, químicos e mineralógicos têm sido pouco estudados.

Ao longo dos últimos 30 anos, o principal método utilizado para determinar as cargas elétricas foi o da titulação potenciométrica (Gillman \& Sumner, 1987; Alleoni \& Camargo, 1994a,b). Nos anos 90s, o método da adsorção de Cs passou a ser empregado na determinação das cargas estruturais em solos de climas: temperado (Chorover et al., 1999), subtropical (Peixoto, 1995) e tropical (Fontes \& Sposito, 1995).

O objetivo deste trabalho foi quantificar as cargas elétricas estruturais e variáveis de Latossolos ácricos, por meio do método da adsorção de $\mathrm{Cs}$, e verificar sua relação com as cargas de um Nitossolo Vermelho, cujo estado de intemperização é menos avançado.

\section{MATERIAL E MÉTODOS}

Foram utilizadas amostras de solos da região norte do Estado de São Paulo, coletadas em duas profundidades: superficial (0-0,2 m) e subsuperficial, no horizonte B, de dois Latossolos Vermelhos acriférricos (LVwf-1 e LVwf-2), dois Latossolos 
Amarelos, um ácrico (LAw) e outro ácriférrico (LAwf), e um Nitossolo Vermelho eutroférrico (NVef), incluído como padrão de solo com material de origem semelhante ao dos outros quatro, mas num estado menos adiantado de evolução.

As amostras de solo foram secas ao ar e passadas em peneiras com malha de $2 \mathrm{~mm}$. A caracterização dos atributos do solo seguiu os métodos descritos em Camargo et al. (1986) e apresentados por Alleoni \& Camargo (1995). Para o presente estudo, obtiveram-se os valores dos seguintes atributos: $\mathrm{pH}$ em água, carbono orgânico, ponto de efeito salino nulo (PESN), CTC, índice de intemperização Ki = $\left(\mathrm{SiO}_{2} / 60\right) /\left(\mathrm{Al}_{2} \mathrm{O}_{3} / 102\right)$, análise granulométrica, análise mineralógica qualitativa, obtida por difração de raios- $\mathrm{X}$, e análise quantitativa, para obter os teores de caulinita e de gibbsita, por meio da análise térmica diferencial (ATD).

Para determinar a carga estrutural, pesaram-se 3,5 g de amostra de solo em tubo de centrífuga de $50 \mathrm{~mL}$. A amostra foi saturada com $24 \mathrm{~g}$ da solução de $\mathrm{CsCl}$ 0,5 mol kg-1 de solução, agitada por $30 \mathrm{~min}$, centrifugada em seguida, por $35 \mathrm{~min}$, com força centrífuga relativa (FCR) de $9.820 \mathrm{x} g$, sendo descartada a solução sobrenadante.

Para reduzir a concentração do excesso de íons, a amostra do solo foi lavada três vezes com $\mathrm{CsCl}$, adicionando-se nas duas primeiras lavagens, $20 \mathrm{~g}$ de $\mathrm{CsCl} 0,1 \mathrm{~mol} \mathrm{~kg}^{-1}$. A amostra foi agitada por $30 \mathrm{~min}$, centrifugada em $9.820 \mathrm{x}$ g, por $35 \mathrm{~min}$, e descartado o sobrenadante. Na terceira lavagem, foram adicionados $20 \mathrm{~g}$ de $\mathrm{CsCl}$ 0,05 mol kg-1, sendo a amostra agitada por $30 \mathrm{~min}$, centrifugada na mesma FCR e descartada a solução sobrenadante. Em seguida, homogeneizou-se a pasta de terra remanescente no tubo de centrífuga e retiraram-se subamostras de 0,5 g das amostras em triplicatas, transferindo-se para cadinhos de porcelana, que foram posteriormente colocados a secar, por $48 \mathrm{~h}$, a $110{ }^{\circ} \mathrm{C}$. Com auxílio dessa umidade, determinou-se $\mathrm{M}_{\text {Pasta do solo seco. }}$.

Após secagem, as amostras foram transferidas para tubos de centrífuga de $50 \mathrm{~mL}$, em triplicatas, $0,35 \mathrm{~g}$ de $\mathrm{M}_{\text {Pasta de solo seco, e suspensas em 3,5 g de }}$ solução de $\mathrm{CsCl} 0,05 \mathrm{~mol} \mathrm{~kg}^{-1}$ a pH 6,0. O ajuste de $\mathrm{pH}$ foi feito com soluções de $\mathrm{CsOH}$ ou de $\mathrm{HCl}$ $0,05 \mathrm{~mol} \mathrm{~kg}^{-1}$. As suspensões foram agitadas, por $60 \mathrm{~min}$, num agitador; em seguida, centrifugadas a $8.820 \times \mathrm{g}$, por $30 \mathrm{~min}$, e descartadas as soluções sobrenadantes.

Para reduzir a concentração de Cs entranhado e para que os complexos de esfera interna entre o íon Cs e as superfícies siloxanas se formassem, as subamostras de solo foram lavadas com $30 \mathrm{~g}$ de etanol $(\mathrm{EtOH})$ a $95 \%(\mathrm{v} / \mathrm{v})$, passando-as em dois banhos ultra-sônicos por 5 min, alternando-se com três agitações num vortex por 10 seg. Centrifugaramse as suspensões etanólicas a 8.820 × g, por $30 \mathrm{~min}$, descartando-se as soluções sobrenadantes. Esta lavagem foi repetida até que nenhum cloreto fosse detectado pelo teste com $\mathrm{AgNO}_{3} 0,01 \mathrm{~mol} \mathrm{~kg}^{-1}$, para, em seguida, as subamostras serem secas a $65^{\circ} \mathrm{C}$, por $48 \mathrm{~h}$, em estufa de circulação de ar forçado.

Para deslocar os íons Cs dos grupos funcionais ionizáveis, as subamostras de solo foram novamente suspensas em $30 \mathrm{~g}$ de $\mathrm{LiCl} 0,01 \mathrm{~mol} \mathrm{~kg}^{-1}$ a pH 6,0. As suspensões foram agitadas por $30 \mathrm{~min}$, utilizando-se agitador horizontal e, em seguida, centrifugadas a $8.820 \times \mathrm{g}$ por $60 \mathrm{~min}$. As soluções sobrenadantes foram transferidas para recipientes plásticos, previamente pesados, e as massas dos extratos de $\mathrm{LiCl}$ foram registradas. Os tubos de centrífuga que continham os solos remanescentes e soluções entranhadas $\left(\mathrm{M}_{\mathrm{T}+\text { pasta do solo seco +sobren }}\right)$ foram pesados a fim de quantificar a massa de solução entranhada $\left(\mathrm{M}_{\mathrm{Li} \text { Entr }}\right)$ (Equação 1).

$$
\begin{gathered}
\mathrm{M}_{\mathrm{Li} \text { Entr }}=\mathrm{M}_{\mathrm{T}+\text { pasta do solo seco }+ \text { sobren }}- \\
-\mathrm{M}_{\mathrm{T}}-\mathrm{M}_{\text {Pasta do solo seco }}
\end{gathered}
$$

Na seqüência, extraiu-se o íon Cs dos sítios com carga estrutural, adicionando-se ao solo remanescente $30 \mathrm{~g}$ da solução de $\mathrm{NH}_{4} \mathrm{OAc}$ $1,0 \mathrm{~mol} \mathrm{~kg}^{-1}$. Agitaram-se as suspensões, por $30 \mathrm{~min}$, em agitador horizontal e, então, centrifugaram-nas a $3.444 \mathrm{x} \mathrm{g}$, por $15 \mathrm{~min}$. As soluções sobrenadantes foram transferidas para frascos plásticos de $250 \mathrm{~mL}$, pré-pesados. Repetiram-se as extrações por três vezes, combinando-se todas as soluções extraídas com $\mathrm{NH}_{4} \mathrm{OAc}$ sendo a massa obtida pesada $\left(\mathrm{M}_{\mathrm{NH} 4 \mathrm{EXT}}\right)$ e armazenada para posterior análise.

As determinações de $\mathrm{Cs}^{+}$nos extratos de $\mathrm{LiCl}$ e de $\mathrm{NH}_{4} \mathrm{OAc}$ foram feitas por espectrofotometria de emissão atômica em chama de ar-acetileno. A curvapadrão foi preparada com base na massa da soluçãoestoque de $\mathrm{CsCl}$ 0,05 mol kg-1 nas mesmas matrizes de soluções de $\mathrm{LiCl}$ e $\mathrm{NH}_{4} \mathrm{OAc}$. Todas as amostras, padrões e diluições devem conter potássio como KCl $\left(5 \mathrm{~g} \mathrm{~kg}^{-1}\right.$ de $\left.\mathrm{K}^{+}\right)$.

A carga variável, $\sigma_{\mathrm{H}},\left(\operatorname{mol}_{\mathrm{c}} \mathrm{kg}^{-1}\right.$ de solo) foi calculada a partir da quantidade de Cs trocável liberada da superfície, após equilíbrio da amostra com $\mathrm{LiCl}$ (equação 2).

$$
-\sigma_{\mathrm{H}}={ }^{\kappa} \mathrm{Cs}(\text { Li sobr }) \quad \times \frac{\text { MLiadic }}{\mathrm{M}_{\text {Pasta do solo seco }}}
$$

na qual $k_{\mathrm{Cs} \text { (Li Sobr) }}$ é a molalidade de $\mathrm{Cs}^{+}$na solução sobrenadante, $\mathrm{M}_{\mathrm{Li} \text { adicion. }}$ é a massa da solução de $\mathrm{LiCl}$ adicionada $\left[\mathrm{M}_{\mathrm{Li}}\right.$ Extrato (massa da solução sobrenadante) $+\mathrm{M}_{\mathrm{Li}}$ Entr (solução entranhada após centrifugação)], e $\mathrm{M}_{\text {Pasta do solo seco é a massa da pasta }}$ seca do adsorvente usado quando do processo da adsorção.

A carga permanente, $\sigma_{\mathrm{O}}\left(\mathrm{mol}_{\mathrm{c}} \mathrm{kg}^{-1}\right.$ de solo) foi calculada como excesso de $\mathrm{Cs}^{+}$retido na carga de superfície específica relativa, seguindo a troca $\mathrm{Li}^{+} \rightarrow \mathrm{Cs}^{+}$(Anderson \& Sposito, 1991) - equação 3: 


$$
-\sigma \mathrm{O}=\mathrm{n}_{\mathrm{CsTot}\left(\mathrm{NH}_{4} \mathrm{Ext}\right)}-\mathrm{M}_{\mathrm{LiEntr}} \times \kappa_{\mathrm{Cs}(\mathrm{Li} \text { sobre })}
$$

em que $\mathrm{n}_{\mathrm{Cs}}$ Tot(NH4 EXT) é o número total de mols de $\mathrm{Cs}^{+}$do solo extraído pelo $\mathrm{NH}_{4} \mathrm{OAc}$ por unidade de massa do adsorvente seco; $\mathrm{M}_{\mathrm{E}(\mathrm{Li})}$ é a massa da solução de LiCl entranhada por unidade de massa do adsorvente seco.

$\mathrm{O}$ termo $\mathrm{n}_{\mathrm{Cs}} \operatorname{Tot}\left(\mathrm{NH}_{4}\right.$ EXT) da equação 4 foi calculado da seguinte forma:

$$
\mathrm{n}_{\mathrm{CSTot}\left(\mathrm{NH}_{4} \mathrm{EXT}\right)}=\kappa_{\mathrm{Cs}_{\mathrm{EXT}}} \times\left(\frac{\mathrm{M}_{\mathrm{NH}_{4} \mathrm{EXT}}}{\mathrm{M} \text { Pastadosolosec o }}\right)
$$

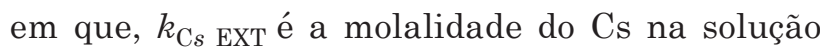

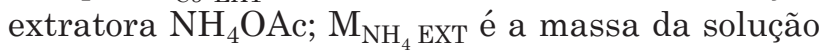
extratora de $\mathrm{NH}_{4} \mathrm{OAc}$.

A densidade da carga total de superfície, $\sigma_{\mathrm{TOTAL}}$ $\left(\mathrm{mol}_{\mathrm{c}} \mathrm{kg}^{-1}\right.$ de solo) foi calculada como a soma de $\sigma_{\mathrm{O}} \mathrm{e}$ $\sigma_{\mathrm{H}},\left(\sigma_{\mathrm{O}}+\sigma_{\mathrm{H}}=\sigma_{\mathrm{TOTAL}}\right)$

\section{RESULTADOS E DISCUSSÃO}

O índice de intemperização é um bom indicador da dessilicatização do material de solo e, conseqüentemente, do estado de intemperização (Bennema et al., 1970), sendo o valores 2,2 admitido como limite superior para os Latossolos. Valores altos indicam predomínio de minerais de argila do tipo $2: 1$, enquanto valores baixos indicam predomínio de óxidos de $\mathrm{Fe}$ e de $\mathrm{Al}$ na fração argila.Os solos ácricos apresentaram valores inferiores a esse limite (variação de 0,60 a 0,99), indicando acentuada dessilicatização e presença de gibbsita. O NVef apresentou Ki 1,74 no horizonte A e 1,89 no B. Dentre os Latossolos ácricos, o LVwf-2 apresentou menor Ki (Quadro 1), enquanto, nas outras amostras, o índice esteve próximo a 1,0.

Os difratogramas da fração argila deferrificada revelaram presença de caulinita e gibbsita em todas as amostras, tendo sido os picos de gibbsitas mais intensos. Na fração argila de alguns Latossolos, predomina a caulinita e, em outros, a gibbsita, e a variação depende do estado de intemperização dos solos (Motchi, 1977). Quanto maior o grau de intemperização, maiores os teores de gibbsita (Rodrigues \& Klamt, 1978). Nos Latossolos ácricos, observaram-se valores de gibbsita bem maiores do que os de caulinita (Quadro 1), diferentemente do que foi encontrado no NVef. Traços de minerais interestratificados e de minerais $2: 1$ individualizados foram identificados em quase todos os solos. A presença de interestratificados tem sido reportada em vários solos brasileiros (Kämpf et al., 1995).

Nas amostras da camada superficial, foram observados os menores valores de PESN, o que está de acordo com Alleoni et al. (2003). Nas amostras

\begin{tabular}{|c|c|c|c|c|c|c|c|c|c|c|c|c|}
\hline Solo & Prof. & $\begin{array}{r}\mathbf{p H} \\
\mathrm{H}_{2} \mathrm{O}\end{array}$ & PESN(1) & $\mathrm{CTC}^{(2)}$ & $\mathbf{C}^{(3)}$ & Silte & Argila & $\mathrm{Ct}^{(4)}$ & $\mathbf{G} \mathbf{b}^{(5)}$ & $\begin{array}{c}2: 1+\text { minerais } \\
\text { mal } \\
\text { cristalizados }^{(6)}\end{array}$ & $\mathbf{K} \mathbf{i}^{(7)}$ & Mineralogia \\
\hline & & & & $\mathrm{mmol}_{\mathrm{c}} \mathrm{kg}^{-1}$ & 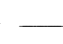 & $\mathrm{g} \mathrm{kg}^{-1} \mathrm{~s}$ & olo - & $-\mathrm{g} \mathrm{kg}^{-1}$ & argila - & & & \\
\hline \multirow[t]{2}{*}{ NVef } & $0-0,20$ & 5,7 & 3,7 & 167 & 26 & 120 & 820 & 297 & 124 & 579 & 1,89 & $\begin{array}{l}\mathrm{C}>\mathrm{G}>\text { VHE cloritizada } \\
\mathrm{Gt} \geq \mathrm{Hm}>\mathrm{Mh}>\text { Magn }\end{array}$ \\
\hline & $0,75-0,95$ & 6,0 & 3,6 & 81 & 07 & 220 & 700 & 289 & 145 & 566 & 1,74 & \\
\hline \multirow[t]{2}{*}{ LVwf-1 } & $0-0,20$ & 6,4 & 3,6 & 93 & 20 & 230 & 600 & 266 & 463 & 271 & 0,93 & $\begin{array}{l}\mathrm{G}>\mathrm{C}>\text { VHE cloritizada } \\
\mathrm{Hm} \geq \mathrm{Gt} \geq \mathrm{Mh}\end{array}$ \\
\hline & $0,80-1,00$ & 5,3 & 5,7 & 22 & 05 & 230 & 640 & 234 & 492 & 274 & 0,87 & \\
\hline \multirow[t]{2}{*}{ LVwf-2 } & $0-0,20$ & 5,1 & 3,4 & 100 & 25 & 120 & 700 & 111 & 449 & 440 & 0,60 & $\begin{array}{l}\mathrm{G}>\mathrm{C}>\mathrm{VHE} \text { cloritizada } \\
\mathrm{Hm} \geq \mathrm{Gt} \geq \mathrm{Mh}\end{array}$ \\
\hline & $1,00-1,50$ & 5,1 & 5,8 & 18 & 06 & 160 & 730 & 134 & 567 & 299 & 0,66 & \\
\hline \multirow[t]{2}{*}{ LAw } & $0-0,20$ & 4,6 & 3,4 & 49 & 16 & 40 & 350 & 235 & 247 & 518 & 0,98 & $\begin{array}{l}\mathrm{G} \geq \mathrm{C}>\text { VHE cloritizada } \\
\mathrm{Gt}>\mathrm{Hm}>\mathrm{Fh}>\mathrm{Qz}\end{array}$ \\
\hline & $0,30-0,70$ & 5,1 & 6,1 & 14 & 06 & 40 & 450 & 209 & 235 & 556 & 0,97 & \\
\hline LAwf & $0-0,20$ & 4,9 & 3,4 & 82 & 24 & 90 & 630 & 183 & 369 & 448 & 0,87 & $\begin{array}{l}\mathrm{G}>\mathrm{C}>\text { VHE cloritizada } \\
\mathrm{Gt}>\mathrm{Hm}>\mathrm{Fh}>\mathrm{Qz}\end{array}$ \\
\hline
\end{tabular}

Quadro 1. Atributos químicos, físicos e mineralógicos em duas profundidades dos solos estudados 
subsuperficiais, tais valores foram maiores, exceto para as amostras do NVef, cujos PESNs foram semelhantes nas duas profundidades, na faixa de 3,6. Destacam-se os elevados valores de PESN no horizonte B dos Latossolos ácricos (de 5,5 a 6,0), decorrente da menor participação de matéria orgânica e do efeito mais pronunciado dos óxidos de $\mathrm{Fe}$ e de $\mathrm{Al}$, principalmente a gibbsita, que naturalmente tende a elevar o PESN dos solos (Hendershot \& Lavkulich, 1978).

Em profundidade, o PESN dos Latossolos foi superior ao $\mathrm{pH}$ do solo, o que lhes conferiu carga líquida positiva nesta camada. Essa reversão de cargas no horizonte B é típica de solos tropicais altamente intemperizados, como os ácricos (Alleoni \& Camargo, 1994a), e isso pode ser constatado nos valores de Ki dos solos (Quadro 1). Ao correlacionar a gibbsita com o PESN destes solos, foi possível fazêlo associando o PESN do Nvef com os Latossolos LVwf 1 e 2, os quais apresentaram correlação positiva $(r=0,83)$, confirmando o efeito da mineralogia na definição das cargas em profundidade.

Para todos os solos, os valores da carga elétrica variável $\left(\sigma_{\mathrm{H}}\right)$ das amostras superficiais foram maiores do que em profundidade, em virtude do maior teor de matéria orgânica. A carga variável correspondeu, em média, a 70 \% da carga total nas camadas superficiais dos solos, com valor mínimo de $55 \%$, para o Nvef, e máximo de $77 \%$, para o LAwf (Quadro 2). Em média, o valor de $\mathrm{ss}_{\mathrm{H}}$ entre os horizontes A e B diminuiu $71 \%$, acompanhando uma queda média de 3,5 vezes no teor de carbono em profundidade. Tais resultados demonstram o importante papel que a matéria orgânica exerce na capa- cidade de troca de íons, corroborando as observações de Raij \& Peech (1972) e Yagi et al. (2003).

O NVef apresentou carga permanente quase cinco vezes superior à dos demais solos, o que pode ser conseqüência da constituição mineralógica, pois os Latossolos continham teores de gibbsita de quatro a cinco vezes maiores que os das amostras do NVef, que, por sua vez, apresentou teores mais elevados de caulinita e minerais $2: 1(\mathrm{VHE})+$ minerais mal cristalizados.

No NVef, no LVw e no Lawf, obtiveram-se correlações positivas entre o índice $\mathrm{Ki}$ e os valores de $\sigma_{0}(r=0,96)$. As amostras do NVef, que é menos intemperizado e por isso tem maior $\mathrm{Ki}$, apresentaram os maiores valores de $\sigma_{0}$. Dentre os Latossolos, o LVwf-1 e o LAwf, com valores próximos de $\mathrm{Ki}$, tiveram cargas permanentes semelhantes e superiores aos das amostras do LVwf-2, cujos valores de $\mathrm{Ki}$ foram os mais baixos. As únicas exceções foram as amostras do LAw, nas quais o Ki esteve próximo de 1,0 (na faixa do LVwf-1 e do LAwf), mas a $\sigma_{0}$ foi bem mais baixa. Isto pode ser explicado pelo menor teor de silte destas amostras (40 $\left.\mathrm{g} \mathrm{kg}^{-1}\right)$, em relação aos demais. Na fração silte, é comum a presença de minerais 2:1 + minerais mal cristalizados, os quais constituem fonte potencial dessas cargas (Lim et al., 1980). Neste solo, a carga permanente de $3,7 \mathrm{mmol}_{\mathrm{c}} \mathrm{kg}^{-1}$ de solo no horizonte $\mathrm{B}$ deveu-se, provavelmente, às cargas originadas de substituição iônica na caulinita, que pode chegar a $20 \mathrm{mmol}_{\mathrm{c}} \mathrm{kg}^{-1}$ de solo, segundo Sposito (1989), e à presença de minerais $2: 1$ que podem estar presentes como impureza na superfície da caulinita, recobrindo parte deste mineral (Lim et al., 1980).

Quadro 2. Cargas elétricas variáveis, permanentes e totais determinadas pelo método da adsorção de Cs em amostras de um Nitossolo e de quatro Latossolos ácricos

\begin{tabular}{|c|c|c|c|c|c|c|}
\hline Solo & Profundidade & $-\sigma_{H}^{(1)}$ & & $-\sigma_{0^{(2)}}$ & & $-\sigma_{\text {TOTAL }}^{(3)}$ \\
\hline & $\mathrm{m}$ & $\mathrm{mmol}_{\mathrm{c}} \mathrm{kg}^{-1}$ de solo & $\% `(4)$ & $\mathrm{mmol}_{\mathrm{c}} \mathrm{kg}^{-1}$ de solo & $\%$ & $\mathrm{mmol}_{\mathrm{c}} \mathrm{kg}^{-1}$ de solo \\
\hline NVef & $\begin{array}{l}0-0,20 \\
0,75-0,95\end{array}$ & $\begin{array}{l}61,8 \pm 0,4 \\
35,8 \pm 0,6\end{array}$ & $\begin{array}{l}55 \\
47\end{array}$ & $\begin{array}{l}51,1 \pm 0,7 \\
41,1 \pm 0,1\end{array}$ & $\begin{array}{l}45 \\
53\end{array}$ & $\begin{array}{r}112,9 \pm 0,3 \\
77,0 \pm 0,6\end{array}$ \\
\hline LVwf-1 & $\begin{array}{l}0-0,20 \\
1,00-1,40\end{array}$ & $\begin{array}{l}28,3 \pm 0,9 \\
17,8 \pm 0,7\end{array}$ & $\begin{array}{l}71 \\
66\end{array}$ & $\begin{array}{r}11,7 \pm 0,2 \\
9,1 \pm 0,3\end{array}$ & $\begin{array}{l}29 \\
34\end{array}$ & $\begin{array}{l}40,0 \pm 0,8 \\
27,0 \pm 0,6\end{array}$ \\
\hline LVwf-2 & $\begin{array}{l}0-0,20 \\
1,00-1,50\end{array}$ & $\begin{array}{l}32,0 \pm 0,5 \\
15,8 \pm 0,1\end{array}$ & $\begin{array}{l}76 \\
73\end{array}$ & $\begin{array}{r}10,4 \pm 0,2 \\
5,9 \pm 0,0\end{array}$ & $\begin{array}{l}24 \\
27\end{array}$ & $\begin{array}{l}42,4 \pm 0,4 \\
21,6 \pm 0,1\end{array}$ \\
\hline LAw & $\begin{array}{l}0-0,20 \\
0,30-0,70\end{array}$ & $\begin{array}{l}14,1 \pm 0,3 \\
10,1 \pm 0,6\end{array}$ & $\begin{array}{l}71 \\
73\end{array}$ & $\begin{array}{l}5,8 \pm 0,2 \\
3,7 \pm 0,2\end{array}$ & $\begin{array}{l}29 \\
27\end{array}$ & $\begin{array}{l}19,9 \pm 0,2 \\
13,8 \pm 0,9\end{array}$ \\
\hline LAwf & $\begin{array}{l}0-0,20 \\
0,60-0,90\end{array}$ & $\begin{array}{l}33,7 \pm 0,3 \\
18,5 \pm 0,3\end{array}$ & $\begin{array}{l}77 \\
69\end{array}$ & $\begin{array}{r}10,6 \pm 0,3 \\
8,4 \pm 0,1\end{array}$ & $\begin{array}{l}23 \\
31\end{array}$ & $\begin{array}{l}44,3 \pm 0,4 \\
27,0 \pm 0,3\end{array}$ \\
\hline
\end{tabular}

${ }^{(1)} \sigma_{\mathrm{H}}=$ adsorção do íon $\mathrm{Cs}^{+}$para os sítios de carga variáveis. ${ }^{(2)} \sigma_{\mathrm{O}}=$ adsorção do íon $\mathrm{Cs}^{+}$para sítios de carga permanente. ${ }^{(3)} \sigma_{\text {total }}=$ adsorção do íon $\mathrm{Cs}^{+}$para sítios de carga total. ${ }^{(4)} \%=$ percentagem em relação ao total.

NVef = Nitossolo Vermelho eutroférrico; LVwf-1 = Latossolo Vermelho acriférrico textura argilosa; LVwf-2 = Latossolo Vermelho acriférrico textura média; LAw = Latossolo Amarelo ácrico; LAwf = Latossolo Amarelo acriférrico. 
Após exame da fração areia muito fina no microscópio óptico, observou-se que os óxidos de Fe recobriam as superfícies de minerais micáceos e nãomicáceos. Esses recobrimentos, segundo Ghabru et al. (1990), reduzem o número de sítios acessíveis à adsorção catiônica da superfície siloxana dos argilominerais.

Os valores da carga total seguiram a tendência das cargas variáveis e das permanentes, isto é, decresceram em profundidade (Quadro 2). Tanto em superfície, quanto em subsuperfície, o NVef apresentou maior $\sigma_{\text {TOTAL }}$, e o LVwf- 1 apresentou a menor.

O método de adsorção de $\mathrm{Cs}$, adotado com grande eficácia na determinação de carga permanente para solos de clima temperado, mostrou-se capaz de detectar significativo teor dessa carga em solos tropicais, principalmente nos Latossolos ácricos, que, apesar de estarem no último nível de intemperização, mantêm cargas estruturais protegidas do desgaste natural.

\section{CONCLUSÕES}

1. A carga variável representou mais que $50 \%$ da carga total dos solos, sendo mais acentuada nos Latossolos ácricos, nos quais ela representou média de $70 \%$ do total.

2. Os Latossolos ácricos exibiram teor significativo de carga permanente, a qual foi atribuída à presença de VHE, mesmo em baixíssimos teores. O NVef apresentou carga permanente cinco vezes maior que os Latossolos ácricos.

\section{AGRADECIMENTOS}

À CAPES, pela bolsa de Doutoramento concedida à primeira autora; ao Dr. Ricardo Trippia G. Peixoto, do IAPAR, pelo apoio técnico e científico durante a realização do trabalho.

\section{LITERATURA CITADA}

ALLEONI, L.R.F \& CAMARGO, O.A. Pontos de efeito salino nulo de Latossolo ácricos. R. Bras. Ci. Solo, 18:175-180, 1994a.

ALLEONI, L.R.F \& CAMARGO, O.A. Potencial elétrico superficial e carga elétrica líquida de latossolos ácricos. R. Bras. Ci. Solo, 18:181-185, 1994b.

ALLEONI, L.R.F. \& CAMARGO, O.A. Óxidos de ferro e de alumínio e a mineralogia da fração argila deferrificada de latossolos ácricos. Sci. Agric., 52:416-421, 1995.
ALLEONI, L.R.F.; ZAMBROSI, F.C.B.; MOREIRA, S.G.; PROCHNOW, L.I. \& PAULETTI, V. Liming and electrochemical properties of an oxisol under no tillage. Sci. Agric. 60:119-123, 2003.

ANDERSON, S.J. \& SPOSITO, G. Cesium adsorption methods for measuring accessible structural surface charge. Soil Sci. Soc. Am. J., 55:1569-1576, 1991.

BENNEMA, J.; JONGERIUS, A. \& LEMOS, R.C. Micromorphology of some oxic and argilic horizons in South Brazil in relation to weathering sequences. Geoderma, 4:333- 358, 1970.

CAMARGO, O.A.; MONIZ, A.C.; JORGE, J.A. \& VALADARES, J.M.A. Métodos de análise química, mineralógica e física de solos do Instituto Agronômico de Campinas. Campinas, 1986. 94p. (IAC-Boletim Técnico, 106)

CHOROVER, J.; DiCHIARO, M.J. \& CHADWICK, O.A. Structural charge ans cesium retention in a chronosequence of tephritic soils. Soil Sci. Soc. Am. J., 63:169-177, 1999.

FONTES, M.P.F. \& SPOSITO, G. Medição da densidade de carga estrutural acessível em Latossolos brasileiros com diferentes mineralogias. In: CONGRESSO BRASILEIRO DE CIÊNCIA DO SOLO, 25., Viçosa, 1995. Anais. Viçosa, Universidade Federal de Viçosa/Sociedade Brasileira de Ciência do Solo, 1995. p.292-294.

FONTES, M.P.F.; CAMARGO, O.A. \& SPOSITO, G. Eletroquímica das partículas coloidais e sua relação com a mineralogia de solos altamente intemperizados. Sci. Agric., 58:627-646, 2001.

GHABRU, S.K.; ARNAUD, R.J.S.T. \& MERMUT, A.R. Association of DCB extractable iron with minerals in coarse soil clays. Soil Sci., 149:112-120, 1990.

GILLMAN, G.P. \& SUMNER, M.E. Surface charge characterization and soil solution composition of four soils from the Southern Piedmont in Georgia. Soil Sci. Soc. Am. J, 5:589-594, 1987.

HENDERSHOT, W.H. \& LAVKULICH, L.M. The use of zero point of charge (ZPC) to assess pedogenic development. Soil Sci. Soc. Am. J, 42:468-472, 1978.

KÄMPF, N.; AZEVEDO, A.C. \& COSTA Jr., M.I. Estrutura básica de argilomineral 2:1 com hidróxi-Al entrecamadas em Latossolo Bruno do Rio Grande do Sul. R. Bras. Ci. Solo, 19:185-190, 1995.

LANDELL, M.G.A.; PRADO, H.; VASCONCELOS, A.C.M.; PERENCIN, D.; ROSSETO, R.; BIDOIA, M.A.P.; SILVA, M.A. \& XAVIER, M.A. Oxisol subsurface chemical attributes related to sugarcane productivity. Sci. Agric., 60:741-745, 2003.

LIM, C.H.; JACKSON, M.L.; KOONS, R.D. \& HEIMKE, P.A. Kaolins: sources of different cation exchange capacities and cesium retention. Clays Clay Miner., 28:223-229, 1980.

MOTCHI, E.P. Características e gênese de uma seqüência de oxissolos no Planalto Central Brasileiro. Porto Alegre, Universidade Federal do Rio Grande do Sul, 1977. 107p. (Tese de Mestrado) 
PEIXOTO, R.T.G. Surface chemical properties of Oxisols derived from Paraná basalt (Brazil). Ohio, Ohio State University, 1995, 188p. (Tese de Doutorado)

RAIJ, B. van \& PEECH, M. Electrochemical properties of some Oxisols and Alfisols of the tropics. Soil Sci. Soc. Am. Proc., 36:587-593, 1972

RODRIGUES, T.E. \& KLAMT, E. Mineralogia e gênese de uma seqüência de solos do Distrito Federal. R. Bras. Ci. Solo, 2:132-139, 1978.

SPOSITO, G. The chemistry of soils. New York, Claredon Press, 1989. 262p.
STUMM, W. \& MORGAN, J.J. Aquatic chemistry. New York, Wiley-Interscience, 1981. 780p.

UEHARA, G. Acric properties and their significance to soil classification. In: INTERNATIONAL SOIL CLASSIFICATION WORKSHOP, 8., Rio de Janeiro, 1986. Proceedings. Rio de Janeiro, Embrapa/ SNLCS, 1988. p.1922.

YAGI, R.; FERREIRA, M.E.; CRUZ, M.C.P. \& BARBOSA, J.C. Organic matter fractions and soil fertility under the influence of liming, vermicompost and cattle manure. Sci. Agric., 60:549-557, 2003. 